\title{
Approximate controllability of nonlocal impulsive neutral integro-differential equations with finite delay
}

\author{
Kamal Jeet ${ }^{1}$ and Dwijendra Pandey ${ }^{2}$ \\ ${ }^{1}$ IIT Roorkee \\ ${ }^{2}$ Indian Institute of Technology Roorkee
}

March 3, 2021

\begin{abstract}
In this paper, we apply the resolvent operator theory and an approximating technique to derive the existence and controllability results for nonlocal impulsive neutral integro-differential equations with finite delay in a Hilbert space. To establish the results, we take the impulsive functions as a continuous function only, and we assume that the nonlocal initial condition is Lipschitz continuous function in the first case and continuous functions only in the second case. The main tools applied in our analysis are semigroup theory, the resolvent operator theory, an approximating technique, and fixed point theorems. Finally, we illustrate the main results with the help of two examples.
\end{abstract}

\section{Hosted file}

Res_prob.pdf available at https://authorea.com/users/399294/articles/511839-approximatecontrollability-of-nonlocal-impulsive-neutral-integro-differential-equations-withfinite-delay 